\title{
Death versus survival: functional interaction between the apoptotic and stress-inducible heat shock protein pathways
}

\author{
Helen M. Beere \\ St. Jude Children's Research Hospital, Department of Immunology, Memphis, Tennessee, USA.
}

\begin{abstract}
Induction of heat shock proteins (Hsps) following cellular damage can prevent apoptosis induced by both the intrinsic and the extrinsic pathways. The intrinsic pathway is characterized by mitochondrial outer membrane permeabilization (MOMP), cytochrome $c$ release, apoptosome assembly, and caspase activation. Hsps promote cell survival by preventing MOMP or apoptosome formation as well as via regulation of Akt and JNK activities. Engagement of the TNF death receptors induces the extrinsic pathway that is characterized by Fas-associated death domain-dependent (FADD-dependent) caspase-8 activation or induction of NF- $\kappa B$ to promote cellular survival. Hsps can directly suppress proapoptotic signaling events or stabilizing elements of the NF- $\kappa \mathrm{B}$ pathway to promote cellular survival.
\end{abstract}

\section{Introduction}

Exposure of cells to potentially damaging stresses such as UV or nutrient withdrawal induces signals able to mediate cell death, or alternatively, survival pathways that allow cells to tolerate and/or to recover from the damage imposed. This paradoxical activation of both pro- and antiapoptotic events in response to the same stimulus ensures that neither aberrant cellular survival nor inappropriate cell death arises and, in doing so, averts the onset and persistence of the pathological state.

The network of heat shock or stress proteins represents an emerging paradigm for the coordinated, multistep regulation of apoptotic signaling events to provide protection from and to facilitate cellular recovery after exposure to damaging stimuli (1-3). Heat shock proteins (Hsps) constitute a highly conserved and functionally interactive network of chaperone proteins, some of which are constitutively expressed and others of which are rapidly induced in response to a variety of chemical, environmental, and physiological stresses. Their collective ability to disaggregate, refold, and renature misfolded proteins offsets the otherwise fatal consequences of damaging stimuli $(4,5)$. This protective function of Hsps has been suggested to reflect their ability to suppress several forms of cell death, including apoptosis.

The Hsp70 and Hsp90 subfamilies are composed of an N-terminal ATPase domain and a C-terminal peptide-binding region that is further characterized by an extreme C-terminal EEVD motif, required for interdomain communication and peptide-binding capacity (6). The C-terminus of Hsp70 is absolutely required for many of its antiapoptotic effects (7). However, the role of the ATPase domain is less clear. Some studies suggest that the ATPase domain, although required, is not sufficient to protect against apoptosis (7), while others indicate that the ATPase domain alone is independently able to inhibit aspects of apoptotic signaling (8).

Nonstandard abbreviations used: DR, death receptor; FADD, Fas-associated death domain; Hsp, heat shock protein; IAP, inhibitor of apoptosis; IKK, IאB kinase; MOMP, mitochondrial outer membrane permeabilization; TRADD, TNFR1-associated death domain.

Conflict of interest: The author has declared that no conflict of interest exists.

Citation for this article: J. Clin. Invest. 115:2633-2639 (2005).

doi:10.1172/JCI26471.

\section{Biochemical characterization of apoptosis}

The primary biochemical signature of apoptosis is the activation of caspases, a family of cysteine proteases with specificity for aspartate residues $(9,10)$. Caspases are produced as inactive zymogens and are broadly characterized according to their mode of activation - (a) "initiator" caspases, including caspase-2, -8, and -9, and (b) "executioner" caspases, exemplified by caspase-3, -6, and -7.

The initiator caspases form active catalytic dimers via association of their long pro-domains with 1 of several adapter molecules with selectivity for individual caspases (11). In contrast, the executioner caspases become catalytically active only after cleavage by the active initiator caspases (11). Once activated, executioner caspases can cleave the initiator caspases. This does not necessarily lead to their activation, but it may serve to stabilize active initiator caspase dimers (11) or render them subject to regulation via the inhibitors of apoptosis (IAPs) $(12,13)$. This hierarchy of caspase activation generates a cascade culminating in executioner-mediated cleavage of substrates including iCAD (14), PARP (15), fodrin (16), p75 (17), actin (18), and focal adhesion kinase (FAK) (19), to display an array of phenotypic characteristics including loss of mitochondrial membrane potential, cell blebbing, condensation and fragmentation of chromatin, and redistribution of lipids in the outer plasma membrane.

The mechanism by which active caspases are generated has led to the categorization of the intrinsic (Figure 1) and extrinsic (Figure 2) apoptotic pathways. The intrinsic pathway is associated with mitochondrial outer membrane permeabilization (MOMP), cytochrome $c$ release, and the activation of procaspase-9. In contrast, the extrinsic pathway can (but does not always) proceed independently of any alteration in mitochondrial function but is instead characterized by the ligation of cell surface death receptors via specific death ligands to generate catalytically active caspase-8. Both pathways result in activation of the executioner caspases and cleavage of their target substrates to induce apoptosis.

\section{Antiapoptotic role of Hsps}

The Hsp27, Hsp70, and Hsp90 subfamilies have been implicated in the protection against apoptosis induced by a variety of stimuli, including chemotherapeutically induced DNA damage, UV radiation (20), polyglutamine repeat expansion (21), death recep- 


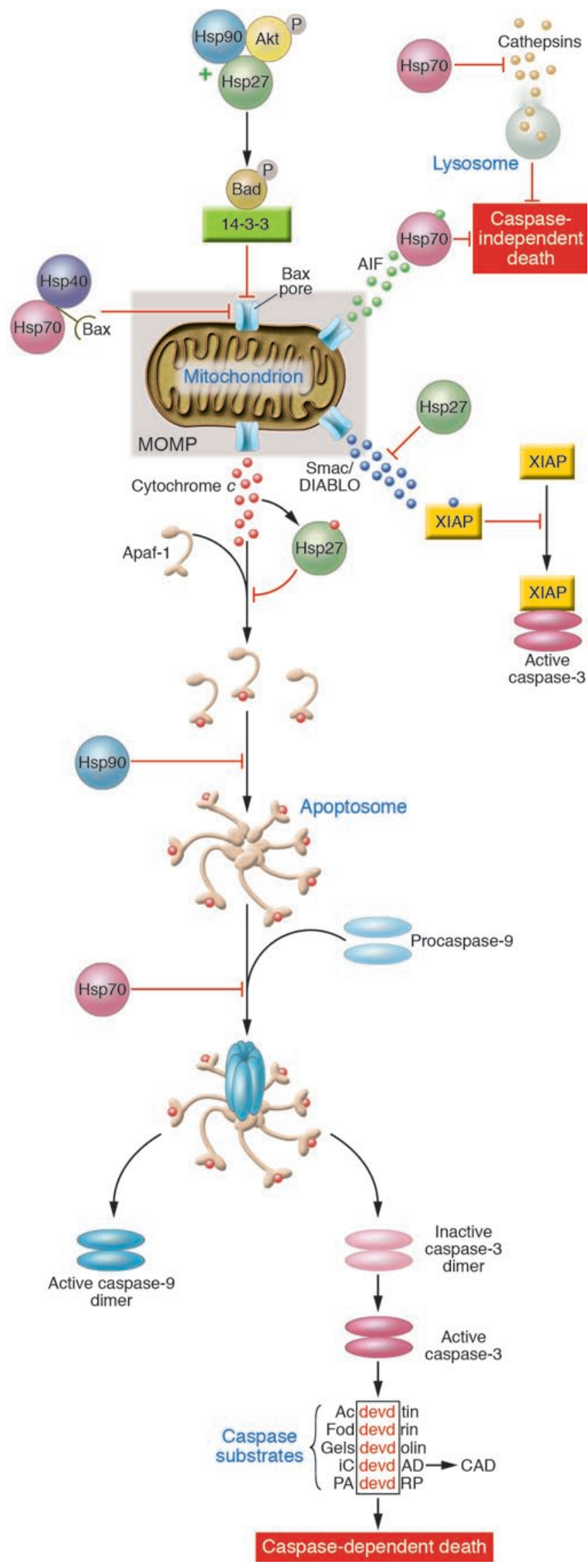

tor ligation $(22,23)$, heat shock $(7,24)$, nutrient withdrawal $(25)$, ceramide (26), reactive oxygen species, ER stresses, proteasome inhibition, and cytoskeletal perturbation. In many cases this inhibition is observed as a suppression of caspase activation and the consequent inhibition of substrate cleavage $(7,27,28)$. Because these events represent the functional end point of upstream apoptotic signals, it is difficult to decipher the likely mechanisms that mediate the survival-promoting effects of the Hsps.

\section{Regulation of mitochondria-mediated apoptosis: the intrinsic pathway}

One defining feature of the intrinsic apoptotic pathway is perturbation of mitochondrial function to induce MOMP and the release of proapoptotic factors that normally reside in the intermembrane space. These include cytochrome $c(29,30)$, the serine protease HtrA2/Omi (31), AIF (32), Smac/DIABLO (33, 34), and endonuclease $\mathrm{G}(35)$ (Figure 1). Although the Bcl-2 family of proteins play a pivotal role in regulating $\operatorname{MOMP}(36,37)$, the physiological significance of each of the intermembrane proteins, once released, remains unclear. This may be attributed to the reported dependence on caspase activity to mediate the release of some of these factors (38), suggesting an ancillary rather than requisite role in cell death.

\section{Regulation of MOMP}

Permeability of the outer mitochondrial membrane is regulated via the opposing activities of the pro- and antiapoptotic members of the Bcl-2 family. The ability of the multidomain proapoptotic $\mathrm{Bcl}-2$ proteins Bax and Bak to induce MOMP is regulated by a number of mechanisms, including changes in localization (Bax ordinarily resides in the cytosol but translocates to the mitochondria following apoptotic stimuli) and alterations in their conformation and oligomeric status. These changes in Bax and Bak, concomitant with their acquisition of permeabilization ability, are likely mediated via the $\mathrm{BH} 3$-only proteins, e.g., Bid, Bim, Bmf, and Bad, each of which is subject to differential regulation according to the type of apoptotic stimulus imposed on the cell $(39,40)$. For example, Bid is cleaved and activated by caspase- 8 following death receptor ligation $(41,42)$; $\operatorname{Bim}(43,44)$ and $\operatorname{Bmf}(45)$ are constitutively sequestered to cytoskeletal components and are released to exert their proapoptotic activities after treatment with drugs such as taxol, or cellular detachment (anoikis), respectively; and the ability of Bad to induce apoptosis is revealed by changes in its phosphorylation status following cytokine withdrawal $(46,47)$.

Hsps can inhibit the mitochondrial release of cytochrome $c$ $(7,48,49)$, consistent with their ability to inhibit caspase activation and substrate cleavage during heat shock-induced death (7). Whether the ability of Hsp70 to block cytochrome $c$ release reflects a direct effect on mitochondrial integrity or the indirect consequences of its intervention at 1 or more points upstream of

\section{Figure 1}

Regulation of the intrinsic pathway by Hsps. Hsps regulate several aspects of the intrinsic apoptotic pathway. These include both direct mediators - e.g., Bax - and indirect regulators - e.g., Akt - of mitochondrial membrane permeabilization to prevent MOMP as well as events downstream of mitochondrial disruption to regulate apoptosome assembly. Caspase-independent cell death may also be affected via Hsp-mediated suppression of AIF activity and inhibition of lysosome permeabilization and cathepsin release. 
MOMP is unclear $(7,48,49)$. However, based on recent studies, the latter seems more likely.

Both Hsp27 and Hsp70 can modulate Bid-dependent apoptosis $(49,50)$. Caspase-8-dependent cleavage of Bid and subsequent Bax/Bak-dependent release of cytochrome $c$ integrates the extrinsic and intrinsic pathways (Figure 2). Hsp70 inhibits TNFinduced release of cytochrome $c$ by suppressing caspase-8-mediated cleavage and activation of Bid, independently of its chaperoning ability (50). Hsp27 can also prevent the translocation of Bid to the mitochondria, which may be related to the ability of Hsp27 to stabilize the cytoskeleton.

In addition, Hsp70 and its co-chaperone Hsp40 prevent the translocation of Bax to the mitochondria during NO-mediated apoptosis, an event that requires both the ATPase and peptidebinding activities of Hsp70 (51) (Figure 1). The co-chaperone proteins play an essential role in regulating the peptide-folding capacity of the Hsps by mediating the interconversion of the ADP- versus ATP-bound states of the Hsps and represent an aspect of Hsp function yet to be fully integrated into our understanding of the role of Hsps in negatively regulating cell death.

MOMP also releases AIF, a factor involved in the induction of caspase-independent cell death $(32,52)$ (Figure 1). Although Hsp70 has not been shown to regulate the release of AIF from mitochondria, it does interact with AIF to prevent the nuclear translocation and pro-death signaling of this molecule $(53,54)$. In a recent study, an AIF-derived protein that retained the Hsp70interacting domain but not its death-inducing activity conferred chemosensitivity to several human cancer cell lines. This was attributed to its ability to sequester and neutralize the antiapoptotic activities of Hsp70 (55). In addition, antisense for Hsp70 can induce a caspase-independent tumor cell death (56) that has been attributed to the ability of Hsp70 to maintain the integrity of lysosomal membranes to prevent cathepsin release into the cytosol (57) (Figure 1).

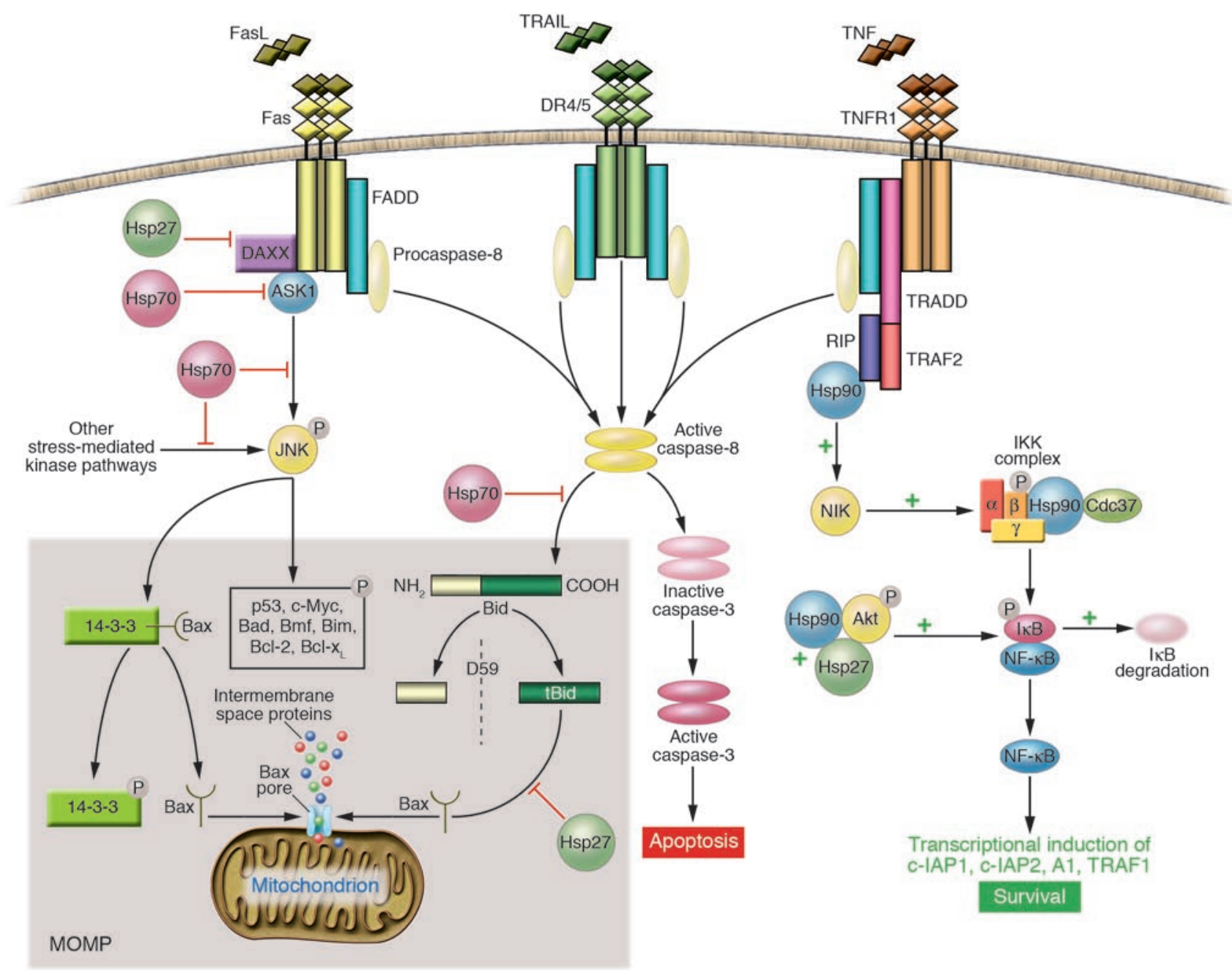

Figure 2

Regulation of the extrinsic pathway by Hsps. Hsps regulate at multiple points within the signaling pathways activated by ligation of a cell surface death receptor by the appropriate ligand. These include the maintenance of prosurvival signals generated via TNF-mediated activation of NF-кB and suppression of proapoptotic signaling events, e.g., JNK activity and Bid cleavage. Integration of the extrinsic and intrinsic pathways is mediated via the caspase-8-mediated cleavage and activation of Bid as well as activation of JNK, which can impact on numerous molecules that regulate mitochondrial integrity (shown in the shaded area). 


\section{Negative regulation of apoptosome assembly}

Cytochrome $c$, integral to the maintenance of mitochondrial respiration, is rapidly released into the cytosol following permeabilization of the outer mitochondrial membrane by the insertion of the multidomain Bcl-2 proteins Bax and Bak $(36,37)$. Once released, cytochrome $c$ induces assembly of the apoptosome, a complex composed of cytochrome $c$, oligomerized Apaf-1 $(58,59)$, and procaspase-9 (60) (Figure 1). Formation of this complex relies on the interaction between Apaf-1 and procaspase- 9 via their respective caspase recruitment domains (CARDs) to generate active caspase- 9 dimers, which then cleave and activate the executioner caspases (60). Apoptosome assembly represents a key regulatory point at which both Hsp70 and Hsp90 can halt the progression of apoptotic signaling, by directly interacting with Apaf-1 to prevent its oligomerization and/or association with procaspase-9 $(61,62)$. Although these observations were recently challenged (48), it is nevertheless intriguing to note that the abilities of Hsp70 and Hsp90 to negatively regulate apoptosome assembly parallel their role in the regulation of protein conformation and/or oligomerization. Hsp27 may also disrupt apoptosome formation via its interaction with and sequestration of cytochrome $c$ after its release from the mitochondria (63).

\section{Hsp-mediated regulation of pro- and antiapoptotic signaling}

Hsps have a profound impact on the activities of several prosurvival signaling cascades, including those mediated by Akt, JNK, and NF- $\kappa B$. By regulating the activities of these upstream signaling molecules, the Hsps can exert an effect on a diverse number of events both distal and proximal to mitochondrial disruption as well as elements of the signaling machinery within the extrinsic pathway (Figure 2).

$\mathrm{PI} 3 \mathrm{~K}$ and its kinase effector protein kinase $\mathrm{B}$ (PKB/Akt) exert a potent prosurvival activity when engaged by cytokines including IGF-1, IL-2, PDGF, and nerve growth factor (64). Withdrawal of these cytokines can elicit an apoptotic cell death that is blocked via Hsps $(25,65)$. Akt can promote cellular survival via 1 of several mechanisms. These include Akt-mediated phosphorylation of Bad that leads to the sequestration of $\mathrm{Bad}$ by the 14-3-3 proteins (66) to prevent its heterodimerization with $\mathrm{Bcl}-2$ or $\mathrm{Bcl}-\mathrm{x}_{\mathrm{L}}$ (46, 47); phosphorylation and inactivation of the forkhead transcription factor FKHRL1 (67), whose target genes include FasL (67), Bim (68), and IGF-1-binding protein (67); and phosphorylation

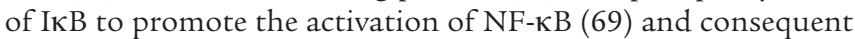
upregulation of several antiapoptotic genes including c-IAP1 (70), c-IAP2 (71), and A1 (72).

Hsp-mediated suppression of cytokine withdrawal-induced apoptosis likely reflects the ability of Hsps to maintain the activity of Akt in the absence of cytokine signaling (73-75). Hsp90, in complex with Cdc37, maintains the stability and activity of Akt by inhibiting its dephosphorylation by PPA2, leading to enhanced cellular survival $(73,76)$. Pharmacological disruption of Hsp90, using geldanamycin, also sensitizes cells to the proapoptotic effects of taxol, a microtubule disruptor, and to the PKC inhibitor UCN-01 (77). This correlates with the ability of geldanamycin to induce the destabilization of Akt and an enhancement of Bax-dependent release of cytochrome $c$ and Smac/DIABLO from mitochondria (78). Hsp27 may also maintain the stability of Akt following apoptotic stimuli that would ordinarily induce its destabilization (75, 79). Hsp27 is phosphorylated by MAPK-activated protein kinase 2
(MAPKAP-K2), an event essential for the ability of Hsp27 to promote survival. Hsp27, recently reported to form a signaling complex with Akt, p38 MAPK, and MAPKAP-K2, is also subject to phosphorylation by Akt. This results in the promotion of an interaction between Akt and Hsp27 and a consequent enhancement of Akt stabilization and cellular survival $(75,79)$.

Activation of the stress-inducible JNK signaling pathway mediates both pro- and antiapoptotic effects depending on the type and duration of the damaging signal as well as cell type (80). Direct targets of JNK activity include Bcl-2 and Bcl- $\mathrm{x}_{\mathrm{L}}$, both of which can be phosphorylated and inactivated by JNK (81-83). JNK may also engage the mitochondrial death pathway via direct phosphorylation and activation of $\operatorname{Bim}$ and $\operatorname{Bmf}(84)$. Recent data also indicate that JNK-mediated phosphorylation of $14-3-3$ proteins can facilitate the translocation of Bax to the mitochondria (85). The proapoptotic $\mathrm{BH} 3$-only protein $\mathrm{Bad}$ is also directly phosphorylated via JNK, although the biological consequence of this event remains unclear (86). This ability of JNK signaling to directly regulate the activities of 1 or more of the Bcl-2 family members is consistent with the failure of UV to induce cytochrome $c$ release in cells lacking JNK (87). Both p53 (88) and c-Myc (89), genes commonly implicated in the etiology of cancer, are regulated by JNK; likewise, both have been shown to directly modulate apoptotic signaling events. At least in the case of p53, this reflects its ability to directly activate $\mathrm{Bax}$ or to bind to and sequester antiapoptotic Bcl- $\mathrm{x}_{\mathrm{L}}$ in a manner analogous to that ascribed to the $\mathrm{BH} 3$-only $\mathrm{Bcl}-2$ proteins, leading to induced cytochrome $c$ release, caspase activation, and apoptotic cell death (90). The ability of JNK to influence mitochondrial integrity is summarized in Figure 2.

Several studies have suggested that the ability of Hsp70 to suppress apoptosis is a direct consequence of its ability to negatively regulate JNK signaling events. Hsp70's negative regulation of JNK activation, and therefore the downstream consequences of its activity, occur independently of its ATPase activity $(24,91)$ via 1 of 2 possible mechanisms: either suppression of its direct phosphorylation and activation by the upstream kinase SEK (92), or inhibition of the stress-induced suppression of JNK dephosphorylation (93).

\section{Antiapoptotic role of Hsps in the extrinsic pathway}

The death receptors belong to a large family of TNF receptor-related members, including TNFR1, Fas, and death receptor 5 (DR5, or TRAILR2), that, when engaged by trimers of their appropriate ligands (TNF, FasL, and TRAIL, respectively), trimerize to elicit an effective and rapid induction of caspase-8-dependent apoptosis that frequently bypasses the requirement for mitochondrial involvement (Figure 2). Following ligand-induced oligomerization of the death receptor, specialized recruitment domains - death domains (DDs) - recruit DD-containing adapter molecules such as Fas-associated death domain (FADD). FADD, in turn, via death effector domains (DEDs), associates with procaspase-8, leading to formation of active caspase- 8 dimers (94). Subsequent autocleavage of procaspase- 8 is observed (11), along with cleavage and activation of procaspase-3 (Figure 2).

Several studies have reported the ability of Hsps to regulate Fas-, TNF-, and TRAIL-dependent pathways of apoptotic cell death $(22,23,95-101)$. However, their underlying mechanisms of action are likely extremely complex, with studies concluding that Hsps can elicit either a positive or a negative effect to promote or to inhibit apoptosis via a number of suggested pathways including suppression of phospholipase A2 activation (99), inhibition of 
the generation of reactive oxygen species (102), and suppression of phosphatase activity (95).

The diversity of death receptors and ligands along with the complexity of their adapter proteins generates an equally complex series of signaling pathways, some of which can mediate cell death, and others of which paradoxically mediate enhanced cellular survival. For example, TNFR1, via a mechanism dependent on TNFR1-associated death domain (TRADD) and FADD, can mediate the activation of caspase- 8 and consequent apoptotic cell death (103-105). Alternatively, the adapter molecule TRADD can mediate the FADD-independent recruitment of a TRAF/RIP kinase complex $(104,105)$, leading to the derepression and nuclear translocation of NF- $\kappa B$. NF- $\kappa B$ is then able to induce the expression of several survival proteins, including c-IAP1 (70), c-IAP2 (71), TRAF1 and TRAF2 (106), and the antiapoptotic Bcl-2 homolog A1 (107).

$\mathrm{NF}-\kappa \mathrm{B}$ is ordinarily sequestered in an inactive cytosolic form via its interaction with IкB. Once phosphorylated by the multi-

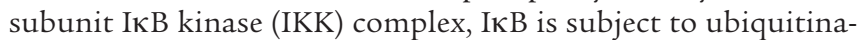
tion and proteasome-dependent degradation, leading to the release of NF-кB. The ability of Hsp27 and Hsp90 to regulate TNF-mediated cellular survival has been linked to their ability to regulate the stability and activity of a number of components of the NF-KB activation pathway, primarily at the level of the IKK complex (108-110). For example, Hsp90, in complex with $\mathrm{Cdc} 37$, a co-chaperone that functions in cooperation with Hsp90 $(111,112)$, binds to the IKK complex via interaction with both the regulatory subunit IKK $\gamma$ (NEMO) and the kinase domains of the catalytic subunits IKK $\alpha$ and IKK $\beta$ (108). This Hsp90-mediated IKK-containing complex appears essential for the efficient TNF-mediated activation of NF- $\mathrm{KB}$ and its survival-promoting effect (108). Accordingly, geldanamycin, a proposed anticancer agent with specificity for Hsp90, confers an enhanced sensitivity of cells to TNF-induced death, consistent with its ability to disrupt Hsp90-containing signaling complexes (113). In contrast, the ability of Hsp27 to interact with both catalytic subunits of the IKK complex, IKK $\alpha$ and IKK $\beta$, the latter of which is enhanced via the TNF-induced activation of MAPK-dependent phosphorylation of Hsp27, leads to the enhanced inhibition of IKK activity,

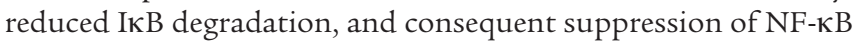
activation (109). These effects of Hsp27 appear to contradict previous findings that it can suppress TNF-induced apoptosis (23, $114)$, but they may simply indicate how poorly we understand the underlying complexity of TNF-induced signaling events and how these might be differentially offset according to the relative expression levels of different Hsps and their predominant mode of antiapoptotic activity. Indeed, a more recent study attributes the survival-promoting activity of Hsp27 to the maintenance of NF- $\kappa \mathrm{B}$ activity via its ability to promote the proteasome-dependent degradation of IкB (110).

The ability of the Hsps to regulate elements of the death receptor-mediated pathways also includes effects at the level of the individual receptor-bound complexes. For example, ligation of the Fas receptor, in addition to leading to the FADD-dependent recruitment and activation of procaspase-8, has also been reported to elicit a FADD-independent pathway via the recruitment of the adapter DAXX, which in turn leads to the activation of the MAPKKK apoptosis signal-regulating kinase 1 (ASK1) and downstream induction of JNK $(115,116)$. Although the role of DAXX in mediating Fas-induced death is controversial (117), the survival-promoting effects of both Hsp27 and Hsp90 have been linked to their ability to regulate the recruitment of DAXX (116) and ASK1 (118) to the Fas signaling complex. Hsp90 can also bind directly to, and stabilize, RIP1 $(119,120)$, thereby providing a more robust signal to induce NF- $\kappa \mathrm{B}$ activation and enhanced cellular survival (113) (Figure 2).

\section{Hsps as pharmacological targets}

In normal cells under nonstressed conditions, the inducible members of the Hsp family are poorly expressed and are only induced after changes in cellular environment or in response to damaging stimuli. In contrast, the expression levels of several members of the Hsp family are significantly elevated in many cancers, and in the case of some tumor types this is linked with poor prognosis and a muted response to chemotherapy (121, 122). It is unclear precisely why Hsp levels are elevated in tumor cells. It may reflect an elevation in the expression of misfolded proteins and a consequent increase in the demand for Hspmediated refolding capacity; or, alternatively, the microenvironment within the tumor, often hypoxic and glucose restricted, may favor the hyperexpression of several Hsps. It is also unclear whether the overexpression of Hsps in tumors simply supports the malignant phenotype (56), or whether they play a more fundamental role in the development and manifestation of the transformed phenotype (123).

Whatever their precise role, the correlation between Hsp overexpression and chemoresistance in many tumor types is likely due, at least in part, to the ability of Hsps to inhibit apoptosis. For that reason, the pharmacological manipulation of Hsp levels likely represents an as-yet underexploited opportunity either to render tumor cells susceptible to the induction of apoptosis by chemotherapeutics and/or UV irradiation or, alternatively, to directly and selectively disrupt their survival.

Geldanamycin and an analog, 17AAG, represent the only Hsptargeted pharmacological agents so far described. These agents bind to and block the nucleotide-binding pocket of Hsp90 and, by doing so, disrupt interactions between Hsp90 and its target proteins $(124,125)$. Targets of Hsp90 that are directly regulated by their ATP-dependent association with Hsp90 include Ras, p53, and Akt, all of which are frequently dysregulated in cancer and play an essential role in the maintenance and progression of the transformed state. Whether this mode of activity might be applied for the disruption of interactions between other Hsp family members and their target proteins has yet to be explored. It does, however, remain a tantalizing possibility that $\mathrm{Hsp}$-targeted pharmacological strategies may herald the advent of a novel approach to rendering tumor cells sensitive to conventional chemotherapies.

\section{Conclusion}

The rationale for a functional interaction between an ancient and highly conserved cellular protective mechanism and a similarly evolutionarily maintained series of pathways to engage cell death seems somewhat intuitive. Just as all species harbor an inducible protective Hsp or stress response, elements of the core apoptotic machinery are likewise found in both uni- and multicellular organisms. It has yet to be determined whether aspects of the Hsp-mediated regulation of apoptotic events observed in mammalian systems are common to lower-order organisms. However, it remains a distinct possibility that to ensure homeostatic regulation of cell number during development and in response to 
damaging events, evolution has incorporated a vital and necessary susceptibility of death-inducing signals to the opposing regulatory effects of the Hsp stress pathway.

\section{Acknowledgments}

The author made every effort to include the key references in each of the areas discussed but apologizes to those whose citations were omitted due to space constraints. H.M. Beere is supported by NIH grants GM066914 and AI47891.

Address correspondence to: Helen M. Beere, St. Jude Children's Research Hospital, Department of Immunology, 332 North Lauderdale Street, Memphis, Tennessee 38105, USA. Phone: (901) 4953470; Fax: (901) 495-3107; E-mail: Helen.Beere@stjude.org.
1. Beere, H.M. 2001. Stressed to death: regulation of apoptotic signaling pathways by the heat shock proteins. Sci. STKE. doi:10.1126/stke.2001.93.re1.

2. Beere, H.M., and Green, D.R. 2001. Stress management: heat shock protein-70 and the regulation of apoptosis. Trends Cell Biol. 11:6-10.

3. Beere, H.M. 2004. "The stress of dying": the role of heat shock proteins in the regulation of apoptosis. J. Cell Sci. 117:2641-2651.

4. Parsell, D.A., and Lindquist, S. 1993. The function of heat-shock proteins in stress tolerance: degradation and reactivation of damaged proteins. Annu Rev. Genet. 27:437-496.

5. Nollen, E.A.A., and Morimoto, R.I. 2002. Chaperoning signaling pathways: molecular chaperones as stress-sensing 'heat shock' proteins. J. Cell Sci. 115:2809-2816.

6. Freeman, B.C., et al. 1995. Identification of a regulatory motif in Hsp70 that affects ATPase activity, substrate binding and interaction with HDJ-1. EMBO J. 14:2281-2292.

7. Mosser, D.D., et al. 2000. The chaperone function of Hsp70 is required for protection against stressinduced apoptosis. Mol. Cell. Biol. 20:7146-7159.

8. Gabai, V.L., et al. 2000. Hsp72-mediated suppression of c-Jun N-terminal kinase is implicated in development of tolerance to caspase-independent cell death. Mol. Cell. Biol. 20:6826-6836.

9. Boatright, K.M., and Salvesen, G.S. 2003. Mechanisms of caspase activation. Curr. Opin. Cell Biol. 15:725-731.

10. Thornberry, N.A. 1998. Caspases: key mediators of apoptosis. Chem. Biol. 5:R97-R103.

11. Boatright, K.M., et al. 2003. A unified model for apical caspase activation. Mol. Cell. 11:529-541.

12. Deveraux, Q.L., et al. 1997. X-linked IAP is a direct inhibitor of cell-death proteases. Nature. 388:300-304.

13. Deveraux, Q.L., and Reed, J.C. 1999. IAP family proteins: suppressors of apoptosis. Genes Dev. 13:239-252.

14. Enari, M., et al. 1998. A caspase-activated DNase that degrades DNA during apoptosis, and its inhibitor ICAD [erratum 1998, 393:396]. Nature. 391:43-50.

15. Lazebnik, Y.A., et al. 1994. Cleavage of poly(ADPribose) polymerase by a proteinase with properties like ICE. Nature. 371:346-347.

16. Martin, S.J., et al. 1995. Proteolysis of fodrin (nonerythroid spectrin) during apoptosis. J. Biol. Chem. 270:6425-6428.

17. Ricci, J.E., et al. 2004. Disruption of mitochondrial function during apoptosis is mediated by caspase cleavage of the p75 subunit of complex I of the electron transport chain. Cell. 117:773-786.

18. Mashima, T., et al. 1997. Actin cleavage by CPP32/apopain during the development of apoptosis. Oncogene. 14:1007-1012.

19. Gervais, F.G., et al. 1998. Caspases cleave focal adhesion kinase during apoptosis to generate a FRNK like polypeptide. J. Biol. Chem. 273:17102-17108

20. Simon, M.M., et al. 1995 . Heat shock protein 70 overexpression affects the response to ultraviolet light in murine fibroblasts. Evidence for increased cell viability and suppression of cytokine release. J. Clin. Invest. 95:926-933.

21. Warrick, J.M., et al. 1999. Suppression of polyglutamine-mediated neurodegeneration in Drosoph- ila by the molecular chaperone HSP70. Nat. Genet. 23:425-428.

22. Clemons, N.J., et al. 2005. Hsp72 inhibits Fasmediated apoptosis upstream of the mitochondria in type II cells. J. Biol. Chem. 280:9005-9012.

23. Mehlen, P., Schulze-Osthoff, K., and Arrigo, A.P. 1996. Small stress proteins as novel regulators of apoptosis. Heat shock protein 27 blocks Fas/ APO-1- and staurosporine-induced cell death. J. Biol. Chem. 271:16510-16514.

24. Mosser, D.D., et al. 1997. Role of the human heat shock protein hsp70 in protection against stressinduced apoptosis. Mol. Cell. Biol. 17:5317-5327.

25. Mailhos, C., Howard, M.K., and Latchman, D.S 1993. Heat shock protects neuronal cells from programmed cell death by apoptosis. Neuroscience. 55:621-627.

26. Ahn, J.H., et al. 1999. Suppression of ceramide-mediated apoptosis by HSP70. Mol. Cell. 9:200-206.

27. Mao, H., et al. 2003. Hsp72 inhibits focal adhesion kinase degradation in ATP-depleted renal epithelial cells. J. Biol. Chem. 278:18214-18220.

28. Garrido, C., et al. 1999. HSP27 inhibits cytochrome c-dependent activation of procaspase-9. FASEB J. 13:2061-2070.

29. Kluck, R.M., et al. 1997. The release of cytochrome c from mitochondria: a primary site for Bcl-2 regulation of apoptosis. Science. 275:1132-1326.

30. Yang, J., et al. 1997. Prevention of apoptosis by $\mathrm{Bcl}-2$ : release of cytochrome $\mathrm{c}$ from mitochondria blocked. Science. 275:1129-1132.

31. Suzuki, Y., et al. 2001. A serine protease, HtrA2, is released from the mitochondria and interacts with XIAP, inducing cell death. Mol. Cell. 8:613-621.

32. Susin, S.A., et al. 1999. Molecular characterization of mitochondrial apoptosis-inducing factor. Nature. 397:441-446.

33. Du, C., et al. 2000. Smac, a mitochondrial protein that promotes cytochrome c-dependent caspase activation by eliminating IAP inhibition. Cell. 102:33-42.

34. Verhagen, A.M., et al. 2000. Identification of DIABLO, a mammalian protein that promotes apoptosis by binding to and antagonizing IAP proteins. Cell. 102:43-53.

35. Li, L., Luo, X., and Wang, X. 2001. Endonuclease G is an apoptotic DNase when released from mitochondria. Nature. 412:95-99.

36. Sharpe, J.C., Arnoult, D., and Youle, R.J. 2004. Control of mitochondrial permeability by Bcl-2 family members. Biochim. Biophys. Acta. 1644:107-113.

37. Willis, S., et al. 2003. The Bcl-2-regulated apoptotic pathway. J. Cell Sci. 116:4053-4056.

38. Arnoult, D., Karbowski, M., and Youle, R.J. 2003. Caspase inhibition prevents the mitochondrial release of apoptosis-inducing factor. Cell Death Differ. 10:845-849.

39. Chao, D.T., and Korsmeyer, S.J. 1998. BCL-2 family: regulators of cell death. Annu. Rev. Immunol. 16:395-419.

40. Marsden, V.S., and Strasser, A. 2003. Control of apoptosis in the immune system: $\mathrm{Bcl}-2, \mathrm{BH} 3$-only proteins and more. Annu. Rev. Immunol. 21:71-105.

41. Gross, A., et al. 1999. Caspase cleaved BID targets mitochondria and is required for cytochrome $c$ release, while BCL-XL prevents this release but not tumor necrosis factor-R1/Fas death. J. Biol. Chem. 274:1156-1163.
42. Luo, X., et al. 1998. Bid, a Bcl2 interacting protein, mediates cytochrome $\mathrm{c}$ release from mitochondria in response to activation of cell surface death receptors. Cell. 94:481-490.

43. O'Connor, L., et al. 1998. Bim: a novel member of the $\mathrm{Bcl}-2$ family that promotes apoptosis. EMBO J. 17:384-395.

44. Puthalakath, H., et al. 1999. The proapoptotic activity of the Bcl-2 family member Bim is regulated by interaction with the dynein motor complex. Mol. Cell. 3:287-296.

45. Puthalakath, H., et al. 2001. Bmf: a proapoptotic $\mathrm{BH}$-only protein regulated by interaction with the myosin $\mathrm{V}$ actin motor complex, activated by anoikis. Science. 293:1829-1832.

46. Zha, J., et al. 1996. Serine phosphorylation of death agonist $\mathrm{BAD}$ in response to survival factor results in binding to 14-3-3 not BCL-X(L). Cell. 87:619-628.

47. del Peso, L., et al. 1997. Interleukin-3-induced phosphorylation of BAD through the protein kinase Akt. Science. 278:687-689.

48. Steel, R., et al. 2004. Hsp72 inhibits apoptosis upstream of the mitochondria and not through interactions with Apaf-1. J. Biol. Chem. 279:51490-51499.

49. Paul, C., et al. 2002. Hsp27 as a negative regulator of cytochrome c release. Mol. Cell. Biol. 22:816-834.

50. Gabai, V.L., et al. 2002. Hsp72 and stress kinase c-jun $\mathrm{N}$-terminal kinase regulate the bid-dependent pathway in tumor necrosis factor-induced apoptosis. Mol. Cell. Biol. 22:3415-3424.

51. Gotoh, T., et al. 2004. hsp70-DnaJ chaperone pair prevents nitric oxide- and CHOP-induced apoptosis by inhibiting translocation of Bax to mitochondria. Cell Death Differ. 11:390-402.

52. Susin, S.A., et al. 1996. Bcl-2 inhibits the mitochondrial release of an apoptogenic protease. J. Exp. Med. 184:1331-1341.

53. Ravagnan, L., et al. 2001. Heat-shock protein 70 antagonizes apoptosis-inducing factor. Nat. Cell Biol. 3:839-843.

54. Gurbuxani, S., et al. 2003. Heat shock protein 70 binding inhibits nuclear import of apoptosisinducing factor. Oncogene. 22:6669-6678.

55. Schmitt, E., et al. 2003. Chemosensitization by a non-apoptogenic heat shock protein 70-binding apoptosis-inducing factor mutant. Cancer Res. 63:8233-8240.

56. Nylandsted, J., et al. 2000. Selective depletion of heat shock protein 70 (Hsp70) activates a tumorspecific death program that is independent of caspases and bypasses Bcl-2. Proc. Natl. Acad. Sci.U. S. A. 97:7871-7876.

57. Nylandsted, J., et al. 2004. Heat shock protein 70 promotes cell survival by inhibiting lysosomal membrane permeabilization. J. Exp. Med. 200:425-435.

58. Zou, H., et al. 1997. Apaf-1, a human protein homologous to C. elegans CED-4, participates in cytochrome c-dependent activation of caspase- 3 . Cell. 90:405-413.

59. Zou, H., et al. 1999. An APAF-1'cytochrome c multimeric complex is a functional apoptosome that activates procaspase-9. J. Biol. Chem. 274:11549-11556.

60. Srinivasula, S.M., et al. 1998. Autoactivation of procaspase- 9 by Apaf-1-mediated oligomerization. Mol. Cell. 1:949-957.

61. Beere, H.M., et al. 2000. Heat-shock protein 70 inhibits apoptosis by preventing recruitment of 
procaspase-9 to the apaf-1 apoptosome. Nat. Cell Biol. 2:469-475.

62. Pandey, P., et al. 2000. Negative regulation of cytochrome c-mediated oligomerization of apaf- 1 and activation of procasapse- 9 by heat shock protein 90. EMBO J. 19:4310-4322.

63. Bruey, J.-M., et al. 2000. Hsp27 negatively regulates cell death by interacting with cytochrome c. Nat. Cell Biol. 2:645-652.

64. Datta, S.D., Brunet, A., and Greenberg, M. 1999. Cellular survival: a play in three Akts. Genes Dev. 13:2905-2927.

65. Mearow, K.M., et al. 2002. Stress-mediated signaling in PC12 cells: the role of the small heat shock protein, Hsp27, and Akt in protecting cells from heat stress and nerve growth factor withdrawal. J. Neurochem. 83:452-462.

66. Dougherty, M.K., and Morrison, D.K. 2004. Unlocking the code of 14-3-3. J. Cell Sci. 117:1875-1884.

67. Brunet, A., et al. 1999. Akt promotes cell survival by phosphorylating and inhibiting a forkhead transcription factor. Cell. 96:857-868.

68. Dijkers, P.F., et al. 2000. Expression of the proapoptotic family member Bim is regulated by the forkhead transcription factor, FKHR-L1. Curr. Biol. 10:1201-1204.

69. Kane, L.P., et al. 1999. Induction of NF-кB by the Akt/PKB kinase. Curr. Biol. 9:601-604.

70. You, M., et al. 1997. ch-IAP1, a member of the inhibitor-of-apoptosis protein family, is a mediator of the antiapoptotic activity of the v-Rel oncoprotein. Mol. Cell. Biol. 17:7328-7341.

71. Chu, Z.L., et al. 1997. Suppression of tumor necrosis factor induced death by inhibitor of apoptosis c-IAP2 is under NF-KB control. Proc. Natl. Acad. Sci. U. S. A. 94:10057-10062.

72. Zong, W.-X., et al. 1999. The pro-survival Bcl-2 homolog Bfl-1/A1 is a direct transcriptional target of NF- $\kappa \mathrm{B}$ that blocks TNF- $\alpha$-induced apoptosis. Genes Dev. 13:382-387.

73. Sato, S., Fujita, N., and Tsuruo, T. 2000. Modulation of Akt kinase activity by binding to Hsp90. Proc. Natl. Acad. Sci. U. S. A. 97:10832-10837.

74. Nakagomi, S., et al. 2003. Expression of the activating transcription factor 3 prevents c-Jun N-terminal kinase-induced neuronal death by promoting heat shock protein 27 expression and Akt activation. J. Neurosci. 23:5187-5196.

75. Rane, M.J., et al. 2003. Heat shock protein 27 controls apoptosis by regulating Akt activation. J. Biol. Chem. 278:27826-27835.

76. Basso, A.D., et al. 2002. Akt forms an intracellular complex with heat shock protein 90 (Hsp90) and $\mathrm{Cdc} 37$ and is destabilized by inhibitors of Hsp90 function. J. Biol. Chem. 277:39858-39866.

77. Jia, W., et al. 2003. Synergistic antileukemic interactions between 17-AAG and UCN-01 interruption of RAF-MEK- and AKT-related pathways. Blood. 102:1824-1832.

78. Nimmanapalli, R., et al. 2003. Regulation of 17 AAG-induced apoptosis: role of Bcl-2, Bcl-XL and Bax downstream of 17-AAG-mediated down regulation of Akt, Raf-1 and Src kinases. Blood. 102:269-275.

79. Rane, M.J., et al. 2001. p38 kinase-dependent MAPKAPK-2 activation functions as 3-phosphoinositide-dependent kinase- 2 for Akt in human neutrophils. J. Biol. Chem. 276:3517-3523.

80. Davis, R.J. 2000. Signal transduction by the JNK group of MAP kinases. Cell. 103:239-252.

81. Fan, M., et al. 2000. Vinblastine-induced phosphorylation of Bcl-2 and Bcl-XL is mediated by JNK and occurs in parallel with inactivation of the Raf-1/ MEK/ERK cascade. J. Biol. Chem. 275:29980-29985.

82. Yamamoto, K., Ichijo, H., and Korsmeyer, S.J. 1999. BCL-2 is phosphorylated and inactivated by an ASK1/Jun N-terminal protein kinase pathway normally activated at G(2)/M. Mol. Biol. Cell.
19:8469-8478.

83. Maundrell, K., et al. 1997. Bcl-2 undergoes phosphorylation by c-Jun $\mathrm{N}$-terminal kinase/stress-activated protein kinases in the presence of the constitutively active GTP binding protein Rac-1. J. Biol. Chem. 272:25238-25242.

84. Lei, K., and Davis, R.J. 2003. JNK phosphorylation of Bim-related members of the Bcl2 family induces Bax-dependent apoptosis. Proc. Natl. Acad. Sci. U. S. A. 100:2432-2437.

85. Tsuruta, F., et al. 2004. JNK promotes Bax translocation to mitochondria through phosphorylation of 14-3-3 proteins. EMBOJ. 23:1889-1899.

86. Yu, C., et al. 2004. JNK suppresses apoptosis via phosphorylation of the proapoptotic Bcl-2 family protein BAD. Mol. Cell. 13:329-340.

87. Tournier, C., et al. 2000. Requirement of JNK for stress-induced activation of the cytochrome cmediated death pathway. Science. 288:870-874.

88. Fuchs, S.Y., et al. 1998. JNK targets p53 ubiquitination and degradation in non-stressed cells. Genes Dev. 12:2658-2663.

89. Noguchi, K., et al. 1999. Regulation of c-Myc through phosphorylation at Ser-62 and Ser-71 by c-Jun N-terminal kinase. J. Biol. Chem. 274:32580-32587.

90. Chipuk, J.E., et al. 2004. Direct activation of Bax by p53 mediates mitochondrial membrane permeabilization and apoptosis. Science. 303:1010-1014.

91. Volloch, V., et al. 1999. ATPase activity of the heat shock protein Hsp72 is dispensable for its effects on dephosphorylation of stress kinase JNK and on heat-induced apoptosis. FEBS Lett. 461:73-76.

92. Park, H.-S., et al. 2001. Hsp72 functions as a natural inhibitory protein of c-Jun $\mathrm{N}$-terminal kinase. EMBO J. 20:446-456.

93. Meriin, A.B., et al. 1999. Protein-damaging stresses activate c-Jun $\mathrm{N}$-terminal kinase via inhibition of its dephosphorylation: a novel pathway controlled by HSP72 [erratum 1999, 19:5235]. Mol. Cell. Biol. 19:2547-2555.

94. Chinnaiyan, A.M., et al. 1995. FADD, a novel death domain containing protein, interacts with the death domain of Fas and initiates apoptosis. Cell. 81:505-512.

95. Liossis, S.N., et al. 1997. Overexpression of the heat shock protein 70 enhances the TCR/CD3- and Fas/Apo-1/CD95-mediated apoptotic cell death in Jurkat T cells. J. Immunol. 158:5668-5675.

96. Creagh, E.M., and Cotter, T.G. 1999. Selective protection by Hsp70 against cytotoxic drug-, but not Fasinduced T-cell apoptosis. Immunology. 97:36-44.

97. Kamradt, M.C., et al. 2005. The small heat shock protein alpha B-crystallin is a novel inhibitor of TRAIL-induced apoptosis that suppresses the activation of caspase-3. J. Biol. Chem. 280:11059-11066.

98. Van Molle, W., et al. 2002. HSP70 protects against TNF-induced lethal inflammatory shock. Immunity. 16:685-695.

99. Jaattela, M. 1993. Overexpression of major heat shock protein hsp70 inhibits tumor necrosis factor-induced activation of phospholipase A2. J. Immunol. 151:4286-4294.

100.Jaattela, M., et al. 1992. Major heat shock protein hsp70 protects tumor cells from tumor necrosis factor cytotoxicity. EMBO J. 11:3507-3512.

101.Galea-Lauri, J., et al. 1996. Increased heat shock protein 90 (hsp90) expression leads to increased apoptosis in the monoblastoid cell line U937 following induction with TNF-alpha and cycloheximide: a possible role in immunopathology. J. Immu nol. 157:4109-4118.

102.Mehlen, P., et al. 1996. Human Hsp27, Drosophila hsp 27 and human $\alpha$ B-crystallin expression-mediated increase in gluathione is essential for the protective activity of these proteins against TNF $\alpha$ induced cell death. EMBO J. 15:2695-2706.

103. Chinnaiyan, A.M., et al. 1996. FADD/MORT is a common mediator of CD95 (Fas/APO1)- and
TNF-receptor-induced apoptosis. J. Biol. Chem. 271:4961-4965

104.Hsu, H., et al. 1996. TNF-dependent recruitment of the protein kinase RIP to the TNF receptor 1 signaling complex. Immunity. 4:387-396.

105. Hsu, H., et al. 1996. TRADD-TRAF2 and TRADDFADD interactions define two distinct TNF receptor signal transduction pathways. Cell. 84:299-308.

106.Wang, C.-Y., et al. 1998. NF-KB antiapoptosis: induction of TRAF1 and TRAF2 and c-IAP1 and c-IPA2 to suppress caspase- 8 activation. Science. 281:1680-1683.

107. Wang, C.Y., et al. 1999. NF-kappaB induces expression of the Bcl-2 homologue A1/Bfl-1 to preferentially suppress chemotherapy-induced apoptosis. Mol. Cell. Biol. 19:5923-5929.

108. Chen, G., Cao, P., and Goeddel, D.V. 2002. TNFinduced recruitment and activation of the IKK complex require Cdc37 and Hsp90. Mol. Cell. 9:401-410.

109.Park, K.-J., Gaynor, R.B., and Kwak, Y.T. 2003. Heat shock protein 27 association with the IKB kinase complex regulates tumor necrosis factor $\alpha$-induced NF-KB activation. J. Biol. Chem. 278:35273-35278.

110.Parcellier, A., et al. 2003. HSP27 is a ubiquitinbinding protein involved in I- $\mathrm{\kappa B} \alpha$ proteasomal degradation. Mol. Cell. Biol. 23:5790-5802.

111. Kimura, Y., et al. 1997. Cdc37 is a molecular chaperone with specific functions in signal transduction. Genes Dev. 11:1775-1785.

112.Septanova, L., et al. 1996. Mammalian p50Cdc37 is a protein kinase-targeting subunit of Hsp90 that binds and stabilizes Cdk4. Genes Dev. 10:1491-1502.

113. Lewis, J., et al. 2000. Disruption of Hsp90 function results in degradation of the death domain kinase, receptor-interacting protein (RIP), and blockage of tumor necrosis factor-induced nuclear factor- $\mathrm{kB}$ activation. J. Biol. Chem. 275:10519-10526.

114. Mehlen, P., et al. 1995. Constitutive expression of human hsp27, drosophila hsp27, or human $\alpha \mathrm{B}$ crystallin confers resistance to TNF- and oxidative stress-induced cytotoxicity in stably transfected murine L929 fibroblasts. J. Immunol. 154:363-374.

115.Chang, H.Y., et al. 1998. Activation of apoptosis signal-regulating kinase (ASK-1) by the adapter protein daxx. Science. 281:1860-1863.

116.Yang, X., et al. 1997. Daxx, a novel fas-binding protein that activates JNK and apoptosis. Cell. 89:1066-1076

117. Torii, S., et al. 1999. Human Daxx regulates Fasinduced apoptosis from nuclear PML oncogenic domains (PODs). EMBO J. 18:6037-6049.

118.Park, H.-S., et al. 2002. Heat shock protein Hsp72 is a negative regulator of apoptosis signal-regulating kinase 1. Mol. Cell. Biol. 22:7721-7730.

119.Devin, A., et al. 2000. The distinct roles of TRAF2 and RIP in IKK activation by TNF-R1: TRAF2 recruits IKK to TNF-R1 while RIP mediates IKK activation. Immunity. 12:419-429.

120.Zhang, S.Q., et al. 2000. Recruitment of the IKK signalosome to the 555 TNF receptor: RIP and A20 bind to NEMO (IKK $\gamma)$ upon receptor stimulation. Immunity. 12:301-311.

121.Jaattela, M. 1999. Escaping cell death: survival proteins in cancer. Exp. Cell Res. 248:30-43.

122.Ciocca, D.R., and Calderwood, S.K. 2005. Heat shock proteins in cancer: diagnostic, prognostic, predictive, and treatment implications. Cell Stress Chaperones. 10:86-103.

123.Volloch, V., and Sherman, M.Y. 1999. Oncogenic potential of Hsp72. Oncogene. 18:3648-3651.

124. Workman, P. 2002. Pharmacogenomics in cancer drug discovery and development: inhibitors of the Hsp90 molecular chaperone. Cancer Detect. Prev. 26:405-410.

125.Neckers, L., and Ivy, S.P. 2003. Heat shock protein 90. Curr. Opin. Oncol. 15:419-424. 